\title{
Antibiotika
}

\section{Antivitamine oder Trojanische Pferde in der Mikrobiologie}

FRANK JANKOWITSCH, JULIA SCHWARZ, VALENTINO KONJIK, CARMEN SCHNEIDER, MATTHIAS MACK

INSTITUT FÜR TECHNISCHE MIKROBIOLOGIE, FAKULTÄT FÜR BIOTECHNOLOGIE, HOCHSCHULE MANNHEIM

Vitamin analogs can be potent antibiotics. We exemplarily study the structural riboflavin (vitamin $B_{2}$ ) analog roseoflavin synthesized by Streptomyces davaonensis to pave the way for the structured analysis of other vitamin analogs yet to be discovered. We investigate the biosynthesis of roseoflavin, its mechanism of action, and the resistance mechanism of the producer. As riboflavin is the most important organic cofactor roseoflavin has a broad impact on the soil microbiome.

DOI: $10.1007 / \mathrm{s} 12268-021-1573-9$

(C) Die Autoren 2021

Antivitamine sind Stoffe, die eine ähnliche Struktur wie Vitamine haben, aber andere physikalische bzw. chemische Eigenschaften. Antivitamine reduzieren oder hemmen die Aktivität bestimmter Enzyme oder RNAMoleküle und damit auch das Wachstum der Zielzellen. Wir untersuchen modellhaft das

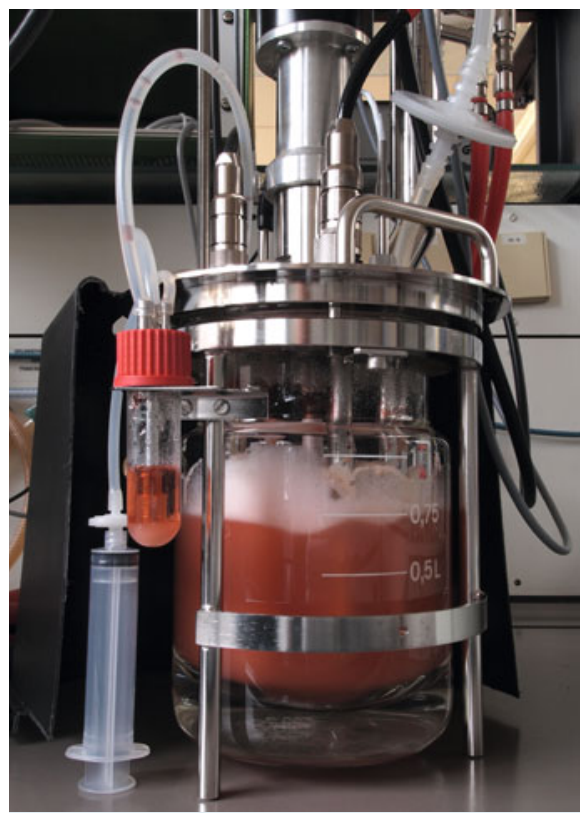

$\Delta$ Abb. 1: Streptomyces davaonensis bildet das rote Antibiotikum Roseoflavin.
Riboflavin-analoge Antivitamin Roseoflavin aus Streptomyces davaonensis, das auch noch von einem zweiten Bakterium, Streptomyces cinnabarinus, gebildet wird. Die Strategie, aus einem lebensnotwendigen Stoff ein Antibiotikum zu bilden, ist clever: Eine gute Aufnahme der antibiotischen Vitaminanaloga durch Vitamintransporter ist garantiert, und damit erreichen diese tückischen Verbindungen als Trojanische Pferde leicht ihr molekulares Ziel. Da die meisten Vitamine an mehreren Stellen biochemisch aktiv sind, haben Antivitamine eine ganze Reihe von Zielmolekülen.

\section{Streptomyces davaonensis bildet das} Antivitamin Roseoflavin

Das Bakterium S. davaonensis wurde von einer japanischen Arbeitsgruppe auf der Suche nach neuen bioaktiven Wirkstoffen aus einer Bodenprobe in der Nähe von Davao City, Philippinen, isoliert [1]. S. davaonensis scheidet auf stärkehaltigen Nährmedien ein wasserlösliches Pigment aus (Abb. 1). Die Arbeitsgruppe isolierte und analysierte das Pigment und nannte es aufgrund der strukturellen Ähnlichkeit zu Riboflavin (Vitamin $\mathrm{B}_{2}$, Abb. 2) und der roten Farbe Roseoflavin. Roseoflavin wirkt auffallend stark antibiotisch gegen Gram-positive Bakterienarten wie Bacillus subtilis oder Staphylococcus aureus, wirkt aber nicht gegen Gram-negative. Klinisch wird Roseoflavin bisher nicht genutzt. Roseoflavin wird für die Selektion mikrobieller Riboflavinüberproduzenten eingesetzt, die zur biotechnologischen Herstellung der wichtigen Feinchemikalie Riboflavin verwendet werden.

\section{Aufnahme und molekulare Wirkung von Roseoflavin}

Unser Labor war das erste, das molekularbiologische und biochemische Arbeiten mit S. davaonensis aufnahm. Schnell fanden wir heraus, warum Roseoflavin nur auf Grampositive Bakterien wirkt: Nur diese besitzen Riboflavintransporter, die auch für die Aufnahme von Roseoflavin sorgen. Nach Aufnahme wird Roseoflavin „aktiviert“ und in die FMN/FAD-Analoga Roseoflavinmononukleotid (RoFMN) bzw. Roseoflavin-Adenin-Dinukleotid (RoFAD) umgewandelt.

Diesen Umbau der Vorstufe (prodrug) Roseoflavin bewerkstelligen Flavokinasen und FAD-Synthetasen. Diese Enzyme sind in allen Lebewesen vorhanden und bilden normalerweise die Enzym-Kofaktoren FMN und FAD aus Riboflavin und ATP. RoFMN und RoFAD binden an Flavoenzyme. Diese Enzyme sind an zahlreichen grundlegenden zellulären Funktionen beteiligt und nur in Kombination mit FMN oder FAD voll aktiv. Wenn Flavoenzyme statt FMN oder FAD RoFMN oder RoFAD gebunden haben, sind sie weniger oder gar nicht mehr aktiv. In einem rekombinanten, Roseoflavin-sensitiven Escherichia coli-Stamm konnten wir beispielsweise zeigen, dass 37 von 38 Flavoenzymen RoFMN oder RoFAD binden und dass dies zu einer signifikanten Reduktion der Aktivität dieser Enzyme führt. Wir konnten darüber hinaus erstmals zeigen, dass FMN-RNA-Schalter durch RoFMN blockiert werden, bzw. dass diese in Bakterien weitverbreiteten genetischen Elemente Zielmoleküle für Antibiotika sein können. FMN-RNA-Schalter regulieren die Biosynthese und den Transport von Riboflavin; werden sie durch RoFMN dauerhaft abgestellt, führt dies zu Riboflavinmangel und 
damit zu reduziertem Wachstum. Merck, USA, entwickelt derzeit ein synthetisches Antiinfektivum (Ribocil) [2], das hemmend auf FMN-RNA-Schalter wirkt, aber keine Vitaminmangelsymptome im Patienten erzeugt.

S. davaonensis besitzt einen ganz speziellen FMN-RNA-Schalter, der zwar noch FMN bindet, aber kein RoFMN, was die Roseoflavinresistenz des Produzenten erklärt. Ganz bestimmte Nukleotide im 285 Nukleotide langen FMN-RNA-Schalter aus $S$. davaonensis, die übrigens auch im FMN-RNA-Schalter des zweiten bekannten Roseoflavinproduzenten S. cinnabarinus vorkommen, sind dabei für die Resistenz verantwortlich. Im Umkehrschluss war die Aufklärung des Resistenzmechanismus ein wichtiger "Beweis“ dafür, dass Roseoflavin tatsächlich auf FMN-RNA-Schalter wirkt [3].

\section{Biosynthese von Roseoflavin}

Wir dachten ursprünglich, dass eine ganze Reihe von Enzymen benötigt würde, um aus Riboflavin Roseoflavin zu bilden bzw. um den Austausch einer Methylgruppe am aromatischen System von Riboflavin durch eine Dimethylaminogruppe zu bewerkstelligen. S. davaonensis löst das Problem jedoch mit wenigen Katalysatoren. Nicht Riboflavin, sondern Riboflavin-5'-phosphat (FMN) ist das Substrat für ein verhältnismäßig kleines Enzym (RosB, 29 kDa), das in einer oxidativen Kaskade zunächst das Schlüsselintermediat 8-Demethyl-8-amino-riboflavin5'-phosphat synthetisiert (Abb. 2, [4]).

Allein wegen RosB hat sich die Untersuchung der Roseoflavinbiosynthese gelohnt. RosB ist das erste bekannte Enzym einer neuen Enzymklasse (mit einem neuen Reaktionsmechanismus), deren Vertreter Oxidoreduktasen, Decarboxylasen und Aminotransferasen in einem sind. Unsere darauffolgende Strukturanalyse von RosB legte nahe, dass RosB aus einem Elektronen-übertragenden Flavodoxin entstanden ist [5]. RosC und RosA komplettieren die Biosynthese von Roseoflavin - insgesamt sind also nur wenige Enzyme nötig, um aus einem Vitamin ein hochwirksames Antibiotikum zu bilden (Abb. 2).

Die Roseoflavin-Biosynthesegene befinden sich auf einem der terminalen Arme des linearen Chromosoms von S. davaonensis in Gesellschaft einer Vielzahl ande- rer Gene des Sekundärstoffwechsels. Noch bilden $\operatorname{ros} B, \operatorname{ros} A$ und $\operatorname{ros} C$ keine Transkriptionseinheit (Abb. 2), und man könnte spekulieren, dass $S$. davaonensis die Kompetenz zur Bildung von Roseoflavin erst spät in der Evolution erworben hat.

Die Sequenzierung des 9,5 Mbp Genoms von $S$. davaonensis und eine darauffolgende Transkriptionsanalyse waren nicht nur für die Aufklärung der Roseoflavinbiosynthese wichtig, sondern ergaben auch, dass $S$. davaonensis mit 17 ungewöhnlich viele funktionelle RiboflavinBiosynthesegene besitzt. S. davaonensis hat demnach eine hohe katalytische Kapazität im Hinblick auf die Bildung von Flavinen, was gut zu einem Produzenten eines Riboflavinanalogons passt. Wir entdeckten u. a. mit RibAB5 ein Enzym, das fünf Mal aktiver ist als alle anderen bekannten RibABs. RibAB5 aus S. davaonensis ist ein schnelles „Eingangsenzym“ der Riboflavinbiosynthese und damit für biotechnologische Anwendungen höchst interessant.

\section{Antibiose des flavinogenen Bakteriums Streptomyces davaonensis}

Riboflavin wird in seiner reduzierten Form (Dihydroriboflavin) von zahlreichen Mikroorganismen, aber auch Pflanzen ausgeschieden, um schlecht lösliches Eisen(III) zu gut löslichem Eisen(II) zu reduzieren (Abb. 3). Neben dieser assimilatorischen Eisenreduktion gibt es im Boden auch die dissimilatorische Eisenreduktion, die darauf abzielt, Reduktionsäquivalente im Rahmen kataboler Prozesse zu entsorgen.

Die Chancen, etwas extrazelluläres Riboflavin zu ergattern, stehen daher für die Mitbewohner im Boden recht gut, was auch daran liegt, dass sich Mikroorganismen gegenseitig durch die Ausscheidung und Aufnahme bestimmter Vitamine (u. a. Riboflavin) unterstützen. Auch wenn wahrscheinlich viele Bodenmikroorganismen Riboflavin selbst synthetisieren können, spart die Aufnahme des Vitamins aus der Umgebung metabolische Energie, schließlich benötigt die Synthese von Riboflavin ein Molekül GTP und zwei Moleküle Ribulose-5-phosphat.

Die Aufnahme von Riboflavin scheint im Boden also normal zu sein was, u. a. erklärt, dass schon neun verschiedene

\section{Hier steht eine Anzeige.}

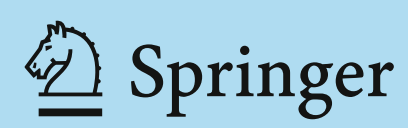




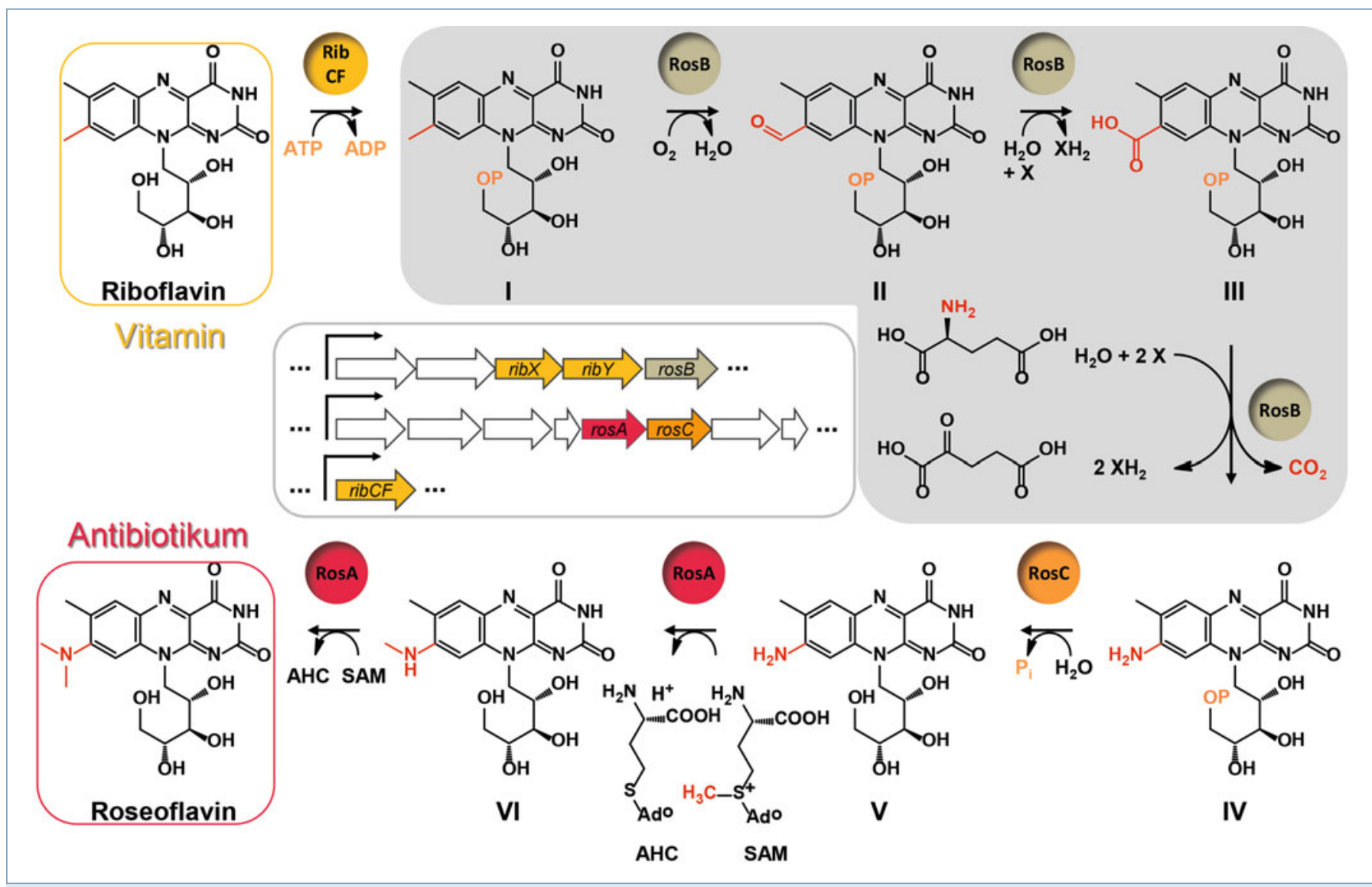

$\Delta$ Abb. 2: Die Biosynthese von Roseoflavin in Streptomyces davaonensis. Die Enzyme sind als Kreise dargestellt. Die Flavokinase RibCF bildet den wichtigen Enzym-Kofaktor Flavinmononukleotid (FMN, I). Das Schlüsselenzym RosB (grau) katalysiert sowohl die Oxidation der Methylgruppe an FMN (I zu III) als auch eine Decarboxylierung bzw. die Übertragung einer Aminogruppe. Die Roseoflavin-Biosynthesegene RosA, RosB und RosC sind auf das lineare Chromosom von $S$. davaonensis verteilt. Die Gene ribXY codieren für ein Riboflavinaufnahmesystem (s. Abb. 3). P: Phosphat.

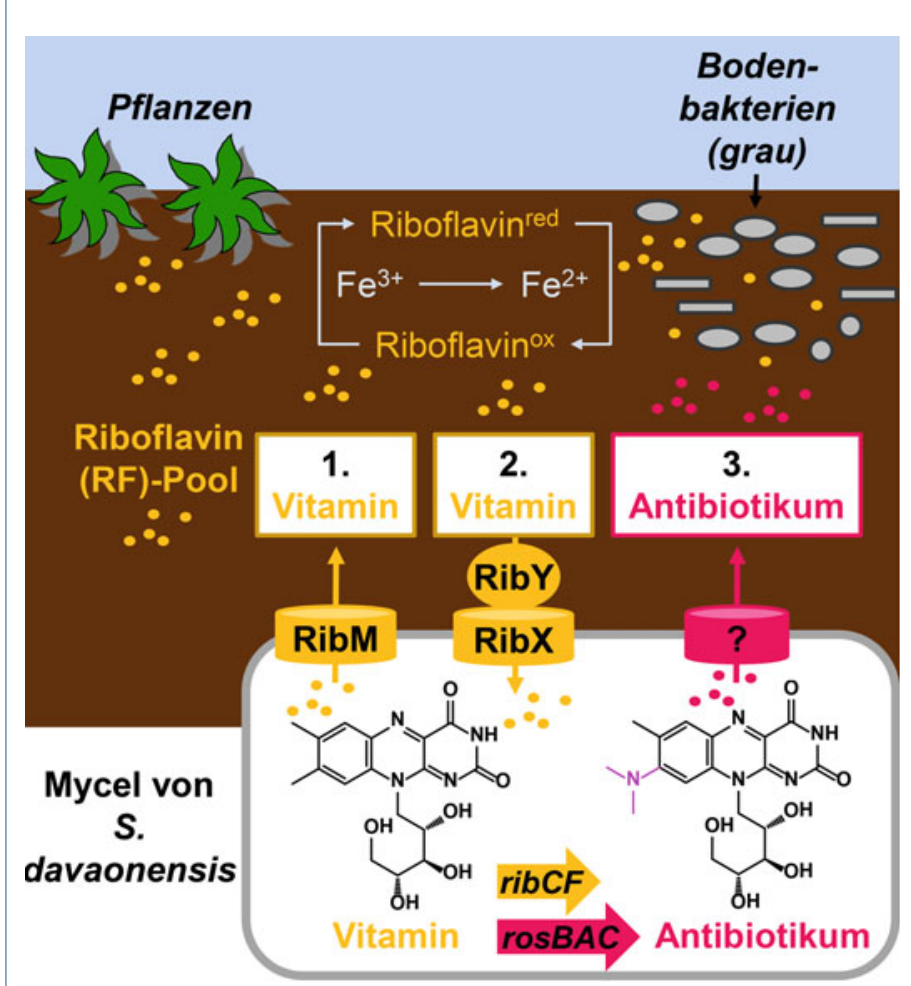

Familien von Riboflavintransportern in verschiedenen Bakterien gefunden wurden. Wir b e z e i c h n e n Roseoflavin gerne als „Trojanisches Pferd" und finden, dass dieses Bild gut zu diesem Riboflavinanalogon passt.
Ahnungslose Nachbarn von S. davaonensis nehmen Roseoflavin mittels Riboflavintransportern auf und gehen zugrunde. S. davaonensis gehört wahrscheinlich zunächst einmal ebenfalls zu den Riboflavinausscheidern (Abb. 3) und füttert die Nachbarn sozusagen an.

Unsere Transkriptomanalysen zeigen, dass S. davaonensis bei Eintritt in die stationäre Phase des Wachstums (RoseoflavinBildungsphase) die Gene eines hocheffizienten Riboflavin-Aufnahmesystems (RibXY) extrem stark exprimiert. S. davaonensis nimmt damit den Nachbarn das Riboflavin
Abb. 3: Durch „Umschalten“ von Riboflavinausscheidung auf Roseoflavinausscheidung verstärkt Streptomyces davaonensis die Wirkung seines Antibiotikums. Riboflavin wird im Zuge der assimilatorischen bzw. dissimilatorischen Eisenreduktion von vielen Bodenmikroorganismen sowie Pflanzen ausgeschieden. Auch das flavinogene Bakterium $S$. davaonensis unterfüttert möglichersweise den Riboflavin-Pool (1.). Bei Eintritt in die stationäre Wachstumsphase nimmt $S$. davaonensis Riboflavin via RibXY wieder auf (2.), wandelt es in Roseoflavin um und scheidet das Antibiotikum wieder aus (3.; der Exporter ist noch unbekannt). 
wieder weg, wandelt es mit den Enzymen RosB, RosC und RosA in Roseoflavin um und scheidet das gebildete Antivitamin wieder aus. In Erwartung eines Vitamins nehmen die Riboflavin-hungrigen Mitbewohner Roseoflavin dann umso bereitwilliger auf. Da Flavine zusammen mit Eisen mit großem Abstand die häufigsten Kofaktoren [6] sind, hat ein toxisches Flavinderivat auch entsprechend großen Einfluss.

\section{Ein verbreitetes System - als Medikament einsetzbar?}

Wir hoffen, dass wir folgende Fragen im Hinblick auf das Vitaminanalogon Roseoflavin noch klären können:

- Welches regulatorische System koordiniert den Stoffwechsel von Riboflavin und Roseoflavin in $S$. davaonensis?

- Welches Protein ist für den Export von Roseoflavin verantwortlich?

- Welche Organismen hemmt Roseoflavin im Boden?

- Gibt es noch weitere natürliche Vitaminanaloga bzw. ist das Antivitaminkonzept noch weiter verbreitet?

- Kann man Roseoflavin als Rückfallmedikament zur Behandlung von z. B. MRSAInfekten klinisch verwenden?

In Zusammenarbeit mit einer australischen Gruppe fanden wir heraus, dass die Behandlung mit Roseoflavin die Proliferation von Malariaerregern hemmt. Für erste in vivoVersuche nutzten wir dabei Plasmodium vinckei vinckei in einem Maus-Infektionsmodell. Die mit Roseoflavin behandelten Mäuse zeigen allerdings die typischen Symptome eines Riboflavinmangels, daher wollen wir als nächstes ein ausgewogeneres Behandlungskonzept entwickeln.

\section{Danksagung}

Die AG Mack bedankt sich für die Förderung bei BMBF, DFG, der Landesstiftung-BadenWürttemberg, der Karl-Völker-Stiftung und der Konanz-Stiftung. Wir bedanken uns bei Jörn Kalinowski für die Sequenzierung und Analyse des Genoms von S. davaonensis und bei Ulrich Ermler für die Strukturanalysen.

\section{Literatur}

[1] Otani S, Takatsu M, Nakano M et al. (1974) Letter: Roseoflavin, a new antimicrobial pigment from Streptomyces. J Antibiot (Tokyo) 27: 86-87

[2] Wang H, Mann PA, Xiao L et al. (2017) Dual-targeting small-molecule inhibitors of the Staphylococcus aureus FMN riboswitch disrupt riboflavin homeostasis in an infectious setting. Cell Chem Biol 24: 576-588
[3] Pedrolli DB, Matern A, Wang J et al. (2012) A highly specialized flavin mononucleotide riboswitch responds differently to similar ligands and confers roseoflavin resistance to Streptomyces davawensis. Nucleic Acids Res 40: 8662-8673 [4] Schwarz J, Konjik V, Jankowitsch F et al. (2016) Identification of the key enzyme of roseoflavin biosynthesis. Angew Chem Int Ed Engl 55: 6103-6106

[5] Konjik V, Brunle S, Demmer U et al. (2017) The crystal structure of RosB: insights into the reaction mechanism of the first member of a family of flavodoxin-like enzymes. Angew Chem Int Ed Engl 56: 1146-1151

[6] Monteverde DR, Gomez-Consarnau L, Suffridge C, Sanudo-Wilhelmy SA (2017) Life's utilization of B vitamins on early Earth. Geobiology 15: 3-18

Funding note: Open Access funding enabled and organized by Projekt DEAL. Open Access: Dieser Artikel wird unter der Creative Commons Namensnennung 4.0 International Lizenz veröffentlicht, welche die Nutzung, Vervielfältigung erlaubt, sofern Sie den/die ursprünglichen Autor(en) und die Quelle ordnungsgemäß nennen, einen Link zur Creative Commons Lizenz beifügen und angeben, ob Änderungen vorgenommen wurden. Die in diesem Artikel enthaltenen Bilder und sonstiges Drittmaterial unterliegen ebenfalls der genannten Creative Commons Lizenz, sofern sich aus der Abbildungslegend nichts anderes ergibt. Sofern das betreffende Material nicht unter der genannten Creative Commons Lizenz steht und die betreffende Handlung nich nach gesetzlichen Vorschriften erlaubt ist, ist für die oben aufgeführt Weiterverwendungen des Materials die Einwilligung des jeweiligen Rechteinhabers einzuholen. Weitere Details zur Lizenz entnehmen Sie bitte der

\section{Korrespondenzadresse:}

Prof. Dr. Matthias Mack

Institut für Technische Mikrobiologie

Fakultät für Biotechnologie

Hochschule Mannheim

Paul-Wittsack-Straße 10

D-68163 Mannheim

m.mack@hs-mannheim.de

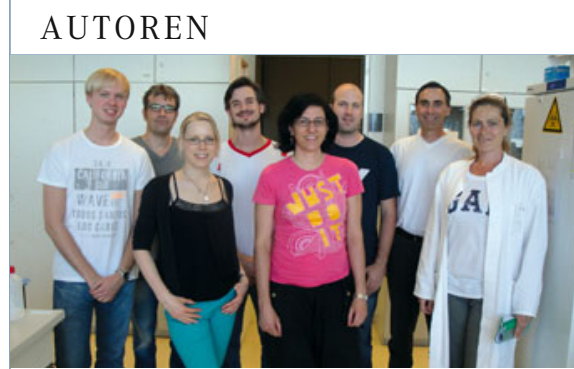

Die AG Mack interessiert sich für Antivitamine und arbeitet darüber hinaus an der Verbesserung mikrobieller Produktionsstämme. Frank Jankowitsch, Julia Schwarz, Valentino Konjik und Carmen Schneider waren als Doktorand/ inn/en im Roseoflavin-Projekt tätig. Matthias Mack leitet das Institut für technische Mikrobiologie und kam im Rahmen seiner Arbeiten zur biotechnologischen Synthese des auch kommerziell wichtigen Riboflavins bei der Firma Hoffmann-La Roche AG in Basel erstmals mit Roseoflavin in Berührung.

\section{Hier steht eine Anzeige.}

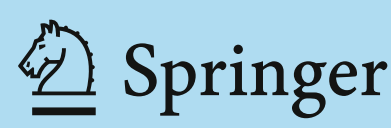

